\title{
7. Geldspenden, Spendenbeträge und freiwilliges Engagement
}

\author{
Nicole Hameister \& Claudia Vogel
}

\section{Kernaussagen}

Mehr als die Hälfte der Wohnbevölkerung Deutschlands spendet Geld. Im Jahr 2014 hat mehr als die Hälfte der Wohnbevölkerung im Alter von 14 und mehr Jahren Geld gespendet (54,4 Prozent).

Zwischen den Bevölkerungsgruppen zeigen sich große Unterschiede in den Spendenquoten. Frauen spenden zu höheren Anteilen als Männer. Mehr ältere Menschen spenden als jüngere, fast drei Viertel der Personen im Alter von 65 und mehr Jahren spenden Geld, aber nur ein knappes Drittel der Personen im Alter von 14 bis 29 Jahren. Zudem spenden Personen mit einem hohen Schulabschluss zu einem deutlich höheren Anteil als Personen mit mittlerer oder mit niedriger Schulbildung.

Beträge von bis zu 100 Euro pro Jahr werden am häufigsten gespendet. Lediglich 2,5 Prozent der Wohnbevölkerung im Alter ab 14 haben in den vergangenen zwölf Monaten mehr als 1.000 Euro gespendet. Personen mit höherem Einkommen spenden nicht nur zu höheren Anteilen Geld, sondern auch höhere Beträge: Bei einem Haushaltsnettoeinkommen von monatlich über 4.000 Euro ist der Anteil der Personen, die spenden, mit 71,5 Prozent überdurchschnittlich hoch. Zudem ist bei diesen Personen der Anteil, der mehr als 1.000 Euro im Jahr spendet, am höchsten.

Im Zeitvergleich spenden heute weniger Personen Geld als vor fünfzehn Jahren. Der Anteil der Personen, die Geld spenden, ist zwischen 1999 und 2014 gefallen (von 63,4 Prozent auf 54,4 Prozent). Insbesondere die jüngeren Altersgruppen spenden heute deutlich seltener als noch vor fünfzehn Jahren. Der Anteil der Personen, die bis zu 100 Euro pro Jahr spenden, sinkt seit 1999 kontinuierlich: Im Jahr 1999 spendeten von allen Personen noch 42,0 Prozent bis zu 100 Euro, 2014 lediglich noch 31,6 Prozent. Bei den hohen Spendenbeträgen zeigt sich Stabilität im Zeitvergleich.

Das Spendenvolumen ist im Zeitvergleich seit 2009 leicht zurückgegangen. Hochgerechnet spendet die Wohnbevölkerung Deutschlands im Jahr 2014 rund 5,9 Milliarden Euro. Im Jahr 2009 betrug das Spendenvolumen noch 6,2 Milliarden. Die Volumen haben wir nach demselben Hochrechnungsverfahren mit identischen Annahmen für beide Zeitpunkte ermittelt.

Personen, die Geld spenden, engagieren sich anteilig häufiger als Personen, die kein Geld spenden. Frauen, die in den vergangenen zwölf Monaten Geld gespendet haben, engagieren sich mit 46,7 Prozent häufiger als Frauen, die nicht gespendet haben (34,7 Prozent), bei Männern liegen die entsprechenden Anteile bei 52,1 Prozent und 39,2 Prozent. 


\subsection{Einleitung}

Viele Menschen in Deutschland spenden nicht nur Zeit im freiwilligen Engagement, sondern auch Geld. In der Regel sind diese Geldspenden zweckgebunden für bestimmte Projekte oder Einrichtungen und unterstützen damit auch häufig das Engagement Anderer. Sie können von den empfangenden Organisationen oder Projekten direkt für ihre Ziele verwendet werden. Laut Sozio-oekonomischem Panel (SOEP) spendeten die Deutschen allein 5,3 Milliarden Euro im Jahr 2009 (Priller \& Schupp 2011). Zwar variieren die Schätzungen des Gesamtspendenvolumens je nach Erhebungsmethode und Datenquelle: Das Statistische Bundesamt weist zum Beispiel geringere jährliche Beträge von lediglich 2,9 Milliarden aus, die auf Basis der Einkommensteuerstatistik 2001 berechnet wurden - und unter anderem deshalb geringer ausfallen, weil nicht alle Einwohnerinnnen und Einwohner einkommensteuerpflichtig sind, aber natürlich trotzdem alle spenden können (Buschle 2006; zu den verschiedenen Datenquellen ausführlich vgl. Priller \& Sommerfeld 2010). Für das Jahr 2010 ist laut Einkommensteuerstatistik ein deutlicher Anstieg des Spendenvolumens auf 6,3 Milliarden Euro festzustellen (Deutsches Zentralinstitut für soziale Fragen (DZI) 2015). Für das Jahr 2014 spricht das Deutsche Zentralinstitut für soziale Fragen sogar von 6,4 Milliarden Euro an Geldspenden für gemeinnützige Zwecke in Deutschland, und zwar auf Basis von Hochrechnungen, die eine Fortschreibung der SOEP-Werte anhand des DZI Spenden-Index darstellen (DZI 2015). Die Gesellschaft für Konsumforschung kommt in ihrer Studie GfK Charity Scope für $2014 \mathrm{zu}$ einem Spendenvolumen von rund 5,0 Milliarden Euro (Gesellschaft für Konsumforschung (GfK) 2015. Allein die Betrachtung des Spendenvolumens insgesamt hilft jedoch nicht weiter, um die Frage zu beantworten, wie das Spendenvolumen zustande kommt. Denn eine große Spendensumme kann sowohl durch viele kleine Spenden als auch durch wenige große Spenden zusammenkommen. Die Summen, die so pro Jahr angesammelt werden, sind jedoch in jedem Fall beträchtlich.

Meist spenden mehr Frauen als Männer, und zwar obwohl Frauen durchschnittlich über geringere Einkommen und Vermögen als Männer verfügen, wie Priller und Schupp (2011) auf Basis des Sozio-oekonomischen Panels berichten. Zudem spenden Ältere zu höheren Anteilen als Jüngere, in diesem Falle übereinstimmend mit vergleichsweise höheren Vermögen und Einkommen der Älteren. Tatsächlich zeigt sich, dass die Spendenquote der Personen im höchsten Einkommensbereich nicht nur am höchsten ist, sondern auch die durchschnittlichen Spendenbeträge am höchsten ausfallen (Priller \& Schupp 2011). Bekkers und Wiepking $(2011 ; 2012)$ zeigen in einer umfassenden Literaturübersicht, dass Bildung ein weiterer zentraler Einflussfaktor für das Spendenverhalten ist, was mit einer vergleichsweise besseren Einkommenssituation Höhergebildeter ebenso einhergehen kann wie mit einem leichteren Zugang $\mathrm{zu}$ Informationen über Spendenbedarfe und Spendenmöglichkeiten. Mit höheren individuellen Ressourcen steigt die Wahrscheinlichkeit, Geld zu spenden.

Mit der Analyse des Freiwilligensurveys 2014 können wir nicht nur eine aktuelle Bestandsaufnahme des Spendenverhaltens der Wohnbevölkerung Deutschlands vornehmen, sondern insbesondere beschreiben, wie sich das Spendenverhalten über einen Zeitraum von fünfzehn Jahren entwickelt hat. Zwei gegensätzliche Annahmen können zur Entwicklung der Spendenanteile formuliert werden: Einerseits ist anzunehmen, dass heute mehr Personen Geld spenden können als jemals zuvor. Das Wohlstandsniveau in Deutschland ist sehr hoch, wie etwa die Arbeitsmarktindikatoren einer Rekordzahl von Beschäftigten und einer geringen Arbeitslosenquote belegen (Statistisches Bundesamt 2015). Die Anlässe, Geld zu spenden sind zudem zahlreich, wie allein die große Zahl von Hilfsorganisationen zeigt, die im Wettbewerb um Spendengelder stehen. Andererseits ist es mög- 
lich, dass heute weniger Personen Geld spenden, da die soziale Ungleichheit in Deutschland zugenommen hat. Viele Menschen haben weniger finanziellen Spielraum und Abstiegsängste etwa bei der unteren Mittelschicht führen möglicherweise dazu, dass weniger Personen etwas abgeben (Lengfeld 2009). Ob dies aber auch bedeutet, dass sie tatsächlich seltener Geld spenden, muss empirisch überprüft werden. Hinweise auf Letzteres gibt der ,World Giving Index' der britischen Charities Aid Foundation: Wilke (2014) berichtet, dass laut diesem Index die Spendenquote in Deutschland von 47 Prozent im Jahr 2012 auf 42 Prozent im Jahr 2013 gesunken sei.

Zum Zusammenhang zwischen Spendenverhalten und freiwilligem Engagement sind gegenläufige Annahmen zu formulieren. Möglicherweise handelt es sich beim Spenden von Geld und dem Spenden von Zeit in Form des freiwilligen Engagements um konkurrierende Tätigkeiten. So könnte es zum Beispiel sein, dass Personen, die bereits Geld gespendet haben, damit dem Bedürfnis zu helfen nachgekommen sind, und keine Zeit mehr spenden wollen, oder umgekehrt, Personen, die eigentlich Zeit spenden wollen, aber keine Zeit haben, stattdessen Geld hierfür aufwenden. Es könnte aber auch sein, dass Geldspenden und freiwilliges Engagement einander ergänzen, etwa weil die Beteiligung im freiwilligen Engagement Gelegenheiten mit sich bringt, auch Geld zu spenden, oder weil ähnliche

\subsection{Datengrundlage}

Die in diesem Kapitel berichteten Befunde basieren auf gewichteten Analysen. Für die Gewichtung wurden in allen Erhebungsjahren dieselben Gewichtungsmerkmale berücksichtigt. Dabei handelt es sich um die Designgewichtung, die sich auf die Auswahlwahrscheinlichkeit einer Person bezieht (Haushaltsgröße, Zahl der Festnetznummern und, nur für das Jahr 2014, auch die Zahl der Mobilfunknummern) und
Motivlagen beide prosozialen Verhaltensweisen gleichermaßen beeinflussen.

In diesem Kapitel werden folgende Forschungsfragen beantwortet:

1. $\mathrm{Zu}$ welchen Anteilen und in welcher Höhe spenden Personen, und welche Unterschiede zeigen sich bezüglich der Spendenquoten und der Spendenbeträge zwischen Frauen und Männern sowie zwischen Alters- und Bildungsgruppen? Da davon auszugehen ist, dass die Spendenbeträge maßgeblich durch die Einkommenssituation bedingt sind, wird zudem untersucht, wie das Spendenverhalten mit dem Haushaltsnettoeinkommen zusammenhängt.

2. Wie hat sich das Spendenverhalten im Zeitvergleich seit 1999 verändert und bei welchen Bevölkerungsgruppen sind diese Änderungen in den Spendenquoten aufgetreten? Wie haben sich die Anteile von Personen im Zeitvergleich verändert, die kleine Beträge von bis zu 100 Euro spenden beziehungsweise die größere Beträge spenden?

3. Wie hat sich das Spendenvolumen zwischen 2009 und 2014 verändert?

4. Welcher Zusammenhang besteht zwischen dem Spendenverhalten und dem freiwilligen Engagement?

Gewichtungsmerkmale, die Abweichungen der Stichprobenverteilung von der Grundgesamtheit in bestimmten Dimensionen ausgleichen (Bundesland, Gemeindegrößenklassen, Geschlecht, Altersgruppen). Von einer Hinzunahme weiterer Gewichtungsmerkmale wie etwa des Bildungsstandes haben wir abgesehen, um die Vergleichbarkeit mit den bisher veröffentlichten Berichten zu erhalten. 
Die Geldspenden sind in der telefonischen Befragung des Deutschen Freiwilligensurveys 2009 und 2014 so erhoben worden:

Manche Menschen leisten gelegentlich oder regelmäßig Geldspenden für soziale oder gemeinnützige Zwecke. Haben Sie in den letzten 12 Monaten solche Spenden geleistet?

Was Personen als Spende bezeichnen - etwa das Überweisen eines Betrages gegen Spendenbescheinigung oder auch den Euro für eine Person, die Obdachlosenmagazine verkauft -, bleibt ihrer subjektiven Einschätzung überlassen. Wenn die Befragten diese Frage bejahen, erfolgte eine Abfrage der Beträge, die gespendet wurden, nach folgenden Stufen:

Waren das in den letzten 12 Monaten insgesamt bis einschließlich 100 Euro oder über 100 Euro?

Wenn über 100 Euro:

Waren es insgesamt bis einschließlich 500 Euro oder über 500 Euro?

Wenn auch über 500 Euro:

Waren es insgesamt bis einschließlich 1.000 Euro oder über 1.000 Euro?

Somit lässt sich berichten, welche Bevölkerungsgruppen $\mathrm{zu}$ welchen Anteilen kleine Beträge von einem Euro bis 100 Euro spenden und zu welchen Anteilen mittlere Beträge von über 100 Euro bis 500 Euro beziehungsweise von über 500 Euro bis 1.000 Euro, und welche Bevölkerungsgruppen zu welchen Anteilen große Beträge von über 1.000 Euro spenden.

Die Ergebnisse zu den Spendenquoten können über die Zeit sehr gut verglichen werden, denn Geldspenden wurden in allen vier Befragungswellen des Freiwilligensurveys mit dieser Frageformulierung erhoben - 1999 und 2004 wurde lediglich eine längere Formulierung durch das zusätzliche Attribut ,karitativ' genutzt:

Manche Menschen leisten gelegentlich oder regelmäßig Geldspenden für karitative, soziale oder gemeinnützige Zwecke. Haben Sie in den letzten 12 Monaten solche Spenden geleistet?

Zwar könnte die etwas kürzere Frageformulierung $\mathrm{zu}$ einem Absinken der Spendenquote beitragen, allerdings hat sich die Spendenquote im Freiwilligensurvey auch zwischen den Wellen 2009 und 2014 verändert, obwohl hier exakt dieselbe Frageformulierung im Telefoninterview verwendet wurde. Bei dieser zu beobachtenden Veränderung ist also davon auszugehen, dass sie reale Veränderungen im Spendenverhalten der Bevölkerung abbildet.

In allen Erhebungswellen wurden Geldspenden zudem mit dem Zeitfenster von zwölf Monaten erhoben. Über den standardisierten Vergleich hinaus hat das Zeitfenster den Vorteil, dass saisonale Unterschiede ausgeglichen sind, die im Spendenverhalten typischerweise vorkommen (zum Beispiel steigt das Spendenaufkommen in der Vorweihnachtszeit und sinkt in den Sommermonaten).

Die Abfrage der Beträge wurde zwischen den Wellen ebenfalls lediglich leicht angepasst, im Jahr 1999 wurde noch nach Deutscher Mark gefragt, seit dem Erhebungsjahr 2004 nach Euro-Beträgen. Die zusätzliche Stufe bei 1.000 Euro wurde erst 2009 eingeführt und bei der Befragung im Jahr 2014 beibehalten (Tabelle 7-1). Seither ist es möglich die größeren Spendenbeträge nochmals zu differenzieren. 2009 und 2014 können wir vier Gruppen unterscheiden. 2004 und früher können wir lediglich drei Gruppen unterscheiden. Die exakte Vergleichbarkeit der nominalen Beträge ist über die Zeit allerdings durch die Währungsumstellung auf Euro und die Inflationsentwicklung über fünfzehn Jahre, welche durchschnittliche Kaufkraftverluste von ein bis zwei Prozent pro Jahr mit sich bringt, beeinträchtigt. 
Tabelle 7-1: Abfrage der Spendenbeträge in den verschiedenen Freiwilligensurvey-Erhebungen

\begin{tabular}{|c|c|}
\hline Welle & Waren das in den letzten 12 Monaten insgesamt... \\
\hline 1999 & $\begin{array}{l}\text { Unter } 200 \text { D-Mark } \\
\text { Über } 200 \text { D-Mark bis } 1.000 \text { D-Mark } \\
\text { Über 1.000 D-Mark }\end{array}$ \\
\hline 2004 & $\begin{array}{l}\text { Bis einschließlich } 100 \text { Euro } \\
\text { Über } 100 \text { Euro bis } 500 \text { Euro } \\
\text { Über } 500 \text { Euro }\end{array}$ \\
\hline 2009 & $\begin{array}{l}\text { Bis einschließlich } 100 \text { Euro } \\
\text { Über } 100 \text { Euro bis } 500 \text { Euro } \\
\text { Über } 500 \text { Euro bis } 1.000 \text { Euro } \\
\text { Über } 1.000 \text { Euro }\end{array}$ \\
\hline 2014 & $\begin{array}{l}\text { Bis einschließlich } 100 \text { Euro } \\
\text { Über } 100 \text { Euro bis } 500 \text { Euro } \\
\text { Über } 500 \text { Euro bis } 1.000 \text { Euro } \\
\text { Über } 1.000 \text { Euro }\end{array}$ \\
\hline
\end{tabular}

Quelle: FWS 2014. Eigene Darstellung (DZA).

\subsection{Geldspenden und Spendenbeträge im Jahr 2014}

Im Jahr 2014 haben 54,4 Prozent der Wohnbevölkerung Deutschlands im Alter ab 14 Jahren mindestens einmal in den vergangenen zwölf Monaten Geld gespendet (Abbildung 7-1a). Die Unterschiede bezüglich der Spendentätigkeit in den verschiedenen Bevölkerungsgruppen sind sehr groß: Im Vergleich von Frauen und Männern im Alter von 14 und mehr Jahren zeigt sich, dass ein größerer Anteil der Frauen (57,2 Prozent) Geld spendet als der Männer (51,5 Prozent). Diese beobachteten Geschlechterunterschiede zeigen sich darüber hinaus in allen Altersgruppen und allen Bildungsgruppen (ohne Abbildung). Da Frauen seltener freiwillig engagiert sind als Männer (siehe Kapitel 3) könnte es sich hierbei um einen Kompensationseffekt dergestalt handeln, dass anstatt Zeit im freiwilligen Engagement $\mathrm{zu}$ spenden, eher Geld gespendet wird. Alternative Erklärungen können ebenfalls nicht ausgeschlossen werden, zum Beispiel engagieren sich Frauen häufiger im sozialen Bereich als Männer, und Spenden werden oft ebenfalls für den sozialen und den humanitären Bereich gesammelt, sodass hier möglicherweise das soziale Engagement durch Geldleistungen ergänzt wird. Zudem engagieren sich Frauen vergleichsweise häufiger im Bereich Kindergarten und Schule, dort könnten vermehrt Schulprojekte gefördert werden, bei denen Spenden eingeworben werden. Außerdem engagieren sich Frauen häufiger im Bereich Kirche und religiöse Vereinigungen, auch hier kommt Geldspenden eine zentrale Funktion zu, da sie in vielen Religionen direkt mit der Religionsausübung in der Gemeinde verbunden sind. 
Abbildung 7-1: Anteile von Personen, die Geld spenden, 2014, a) gesamt, nach Geschlecht und nach Alter, b) nach Bildung

a) gesamt, nach Geschlecht und nach Alter

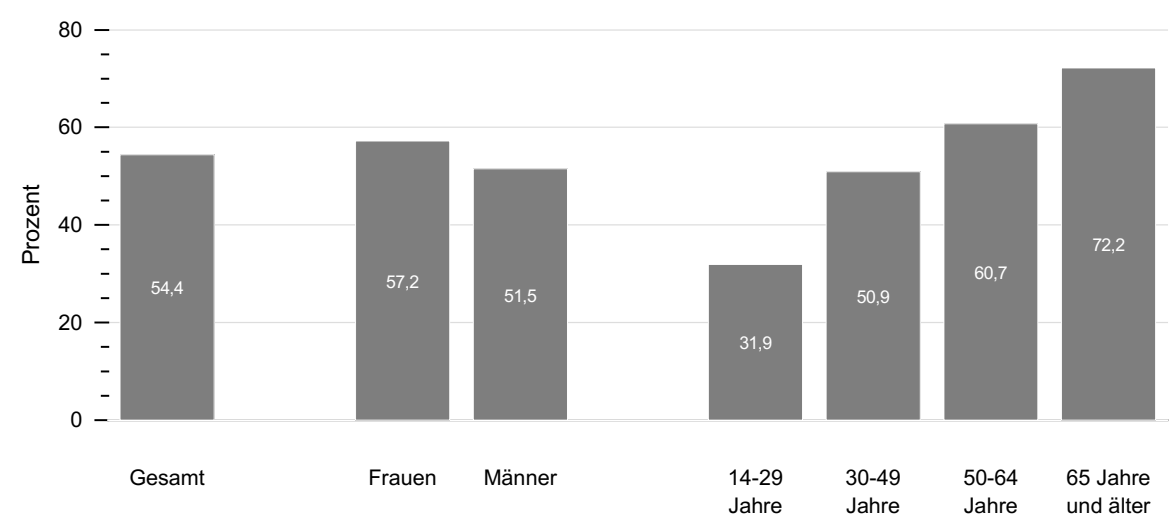

\section{b) nach Bildung}

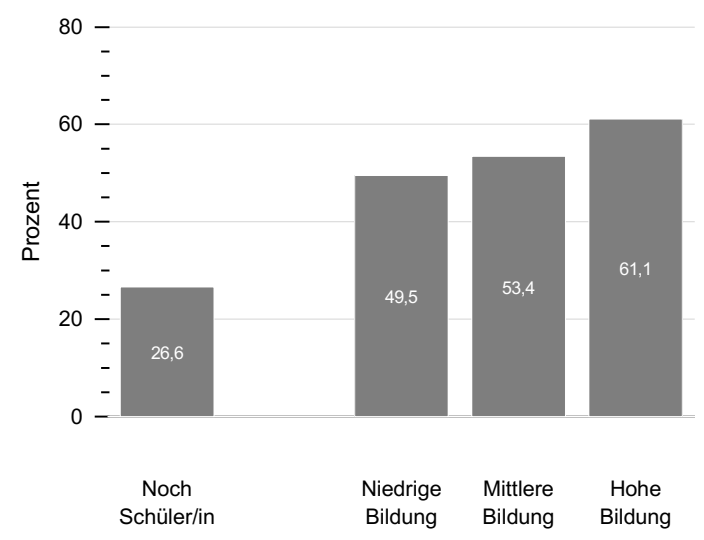

Quelle: FWS 2014, gewichtet, eigene Berechnungen (DZA). Basis: Alle Befragten $(n=28.574)$.

Der Unterschied im Anteil der Geldspenderinnen im Vergleich zum Anteil der Geldspender ist jedoch vergleichsweise gering im Gegensatz zu den großen Unterschieden, die sich zwischen den vier in unserer Analyse betrachteten Altersgruppen zeigen. Mit Abstand am häufigsten spenden Personen, die 65 Jahre oder älter sind (Abbildung 7-1a): 72,2 Prozent von ihnen haben in den vergangenen 12 Monaten etwas gespendet. Auch bei den 50- bis 64-Jährigen sind die
Anteile der Spenderinnen und Spender überdurchschnittlich hoch. In dieser Altersgruppe haben 60,7 Prozent in den vergangenen zwölf Monaten mindestens einmal Geld gespendet.

Betrachten wir die unterschiedlichen Anteile von Spenderinnen und Spendern in den vier Bildungsgruppen, kommen wir zu einer ähnlichen Einschätzung. Am seltensten haben Kinder, Jugendliche und junge Erwachsene Geld gespendet, die noch in Schule oder Ausbildung 
sind (Abbildung 7-1b): Lediglich 26,6 Prozent der Schülerinnen und Schüler haben in den vergangenen zwölf Monaten gespendet (Abbildung 7-1b). Zudem zeigt sich ein deutlicher Bildungsunterschied bei Personen mit abgeschlossener Schulbildung. Am häufigsten spenden diejenigen Geld, die hochgebildet sind (61,1 Prozent). Bei den Personen mit mittlerer Bildung spenden 53,4 Prozent und bei den Personen mit niedriger Bildung ist der Anteil unterdurchschnittlich (49,5 Prozent).

Die Unterschiede zwischen den Bildungsgruppen weisen zwar in allen Altersgruppen in dieselbe Richtung - wer höher gebildet ist, spendet häufiger -, allerdings scheint hier die Lebensphase einen moderierenden Einfluss zu haben. Niedriggebildete im Alter von 65 Jahren und mehr Jahren spenden $\mathrm{zu}$ einem deutlich höheren Anteil als Niedriggebildete im Alter von 14 bis 29 Jahren oder im Alter von 30 bis 49 Jahren. Zudem spenden niedriggebildete Personen im Alter von 65 und mehr Jahren auch zu einem höheren Anteil als die Hochgebildeten in den jüngeren Altersgruppen (ohne Abbildung). Dies könnte damit zusammenhängen, dass die Einkommenssituation im Alter nicht nur von individuellen Faktoren wie dem erreichten Schulabschluss, sondern vor allem von der Haushaltskonstellation abhängig ist, da die Alterssicherung sich aus den individuellen Renten-, Miet- und Kapitaleinkünften der Ehepaare ergibt (Motel-Klingebiel \& Vogel 2013).

Insbesondere diese Altersgruppen- und Bildungsunterschiede sind auch beträchtlich bezüglich der gespendeten Geldbeträge: Mit 31,3 Prozent werden am häufigsten Beträge von bis zu 100 Euro pro Jahr gespendet (Abbildung 7-2a). Hohe Beträge von 501 bis 1.000 Euro und sehr hohe Beträge von über 1.000 Euro werden lediglich von einer jeweils sehr kleinen Bevölkerungsgruppe gespendet (3,0 Prozent beziehungsweise 2,5 Prozent). Obwohl Frauen anteilig häufiger
Geld spenden als Männer, kommt der höhere Spendenanteil bei Frauen vor allem durch den höheren Anteil von Spenden bis zu 100 Euro zustande: 35,1 Prozent der Frauen, aber nur 28,0 Prozent der Männer spenden diese kleinen Beträge im Zeitraum von zwölf Monaten.

Sehr hohe Beträge von über 1.000 Euro spenden am häufigsten Personen der Altersgruppe 65 Jahre und älter (4,7 Prozent) beziehungsweise 50 - bis 64-Jährige (3,0 Prozent). Dies könnte auch dadurch begünstigt sein, dass die durchschnittlichen monatlichen Einkommen der 50- bis 64-Jährigen über dem Bevölkerungsdurchschnitt liegen. In der Lebenslaufperspektive betrachtet ist das Erwerbseinkommen zum Ende der Erwerbskarriere am höchsten und auch Kapitalerträge aufgrund angesparter Vermögen können reichlicher fließen als in jungen Jahren. Rentnerinnen und Rentner verfügen darüber hinaus heute über überdurchschnittlich hohe Vermögen in Deutschland, allerdings sind ihre Alterseinkommen meist geringer als die früheren Erwerbseinkommen. Der Hauptgrund dafür ist, dass die Beträge der Rentenzahlungen aus der gesetzlichen Rentenversicherung unter den Erwerbseinkommen liegen und Einkommen aus privaten Altersvorsorgeprodukten nicht sehr weit verbreitet sind (Motel-Klingebiel \& Vogel 2013). Jüngere Personen verdienen meistens nicht nur weniger während der Ausbildung und dem Berufsstart, sondern sie haben auch deutlich höhere Konsumausgaben, wenn etwa eine Familie gegründet wird, eine Immobilie gekauft und eingerichtet werden muss und neben der eigenen Altersvorsorge auch der Lebensunterhalt und die Ausbildung der Kinder finanziert werden soll. Personen im Alter von 14 bis 29 Jahren spenden nicht nur seltener, sondern insbesondere meist geringe Beträge von bis zu 100 Euro. In dieser Altersgruppe verfügt ein Teil der Jugendlichen sowie der jungen Erwachsenen noch nicht über ein eigenes Einkommen. 
Abbildung 7-2: Anteile von Personen, die Geld spenden, 2014, differenziert nach gespendeten Beträgen, a) gesamt, nach Geschlecht und nach Alter, b) nach Bildung

a) gesamt, nach Geschlecht und nach Alter

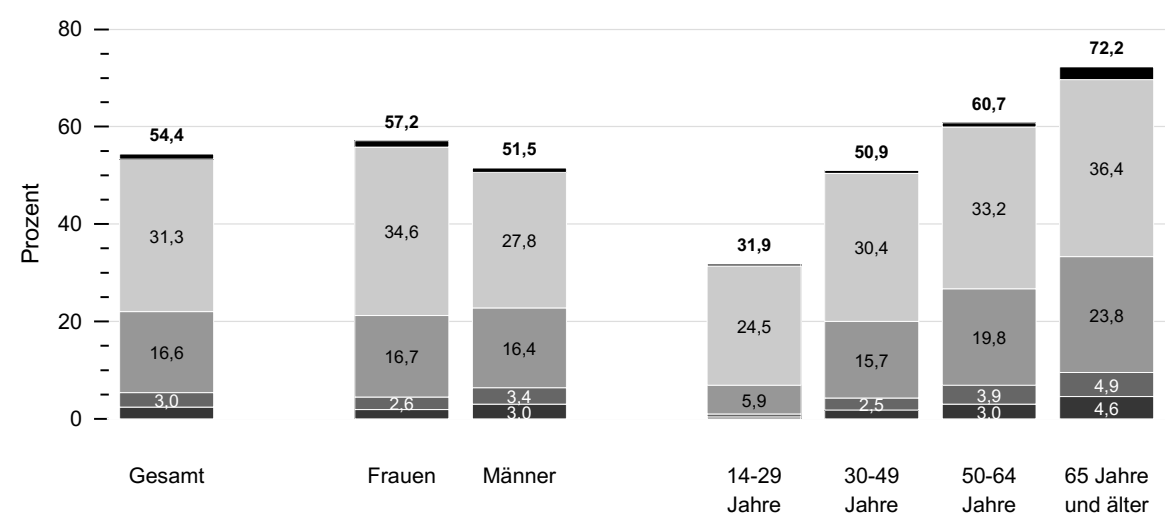

\section{b) nach Bildung}

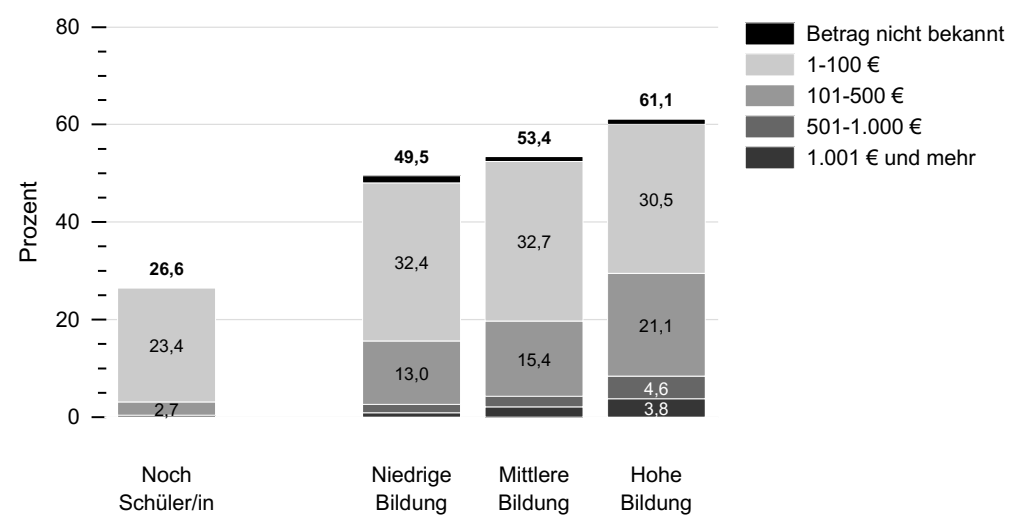

Quelle: FWS 2014, gewichtet, eigene Berechnungen (DZA). Basis: Alle Befragten ( $n=28.300$ ).

Insgesamt spenden Personen sicherlich nicht nur, weil sie es sich aufgrund ihrer ökonomischen Situation leisten können, etwas abzugeben, sondern auch, weil sie wohl in erster Linie tatsächlich helfen und durch ihren Beitrag etwas bewegen wollen. Dennoch schlägt sich die vergleichsweise bessere Einkommens- und Vermögensposition der Hochgebildeten vor allem in einem überdurchschnittlichen Anteil von Personen nieder, die große Beträge von über 1.000 Euro spenden (3,8 Prozent). Besonders deutlich wird die Abhängigkeit der Spendenbeträge vom Einkommen, wenn die gespendeten Beträge in Bezug zum Haushaltsnettoeinkommen gesetzt werden. 
Abbildung 7-3: Anteile von Personen, die Geld spenden, differenziert nach gespendeten Beträgen, 2014, nach Haushaltsnettoeinkommen

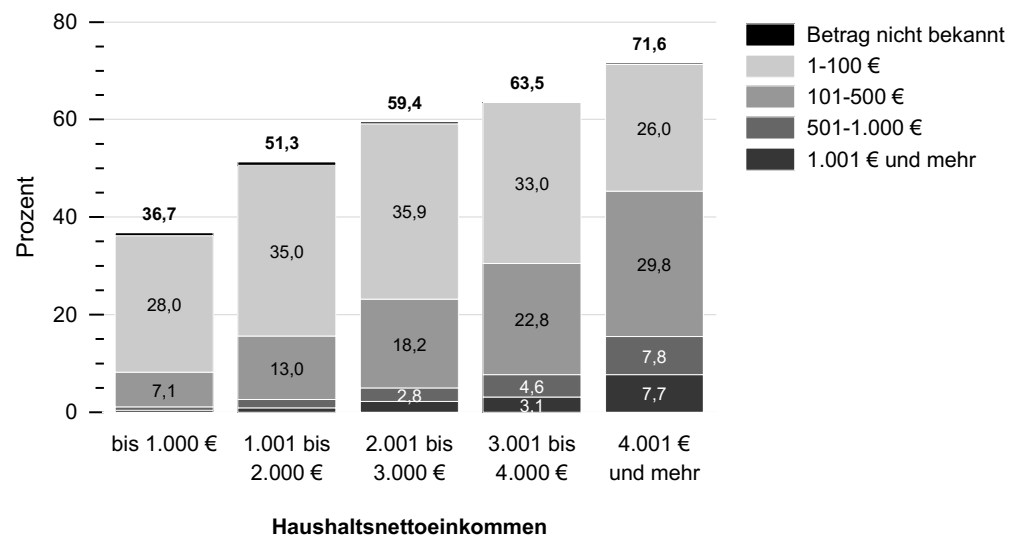

Quelle: FWS 2014, gewichtet, eigene Berechnungen (DZA). Basis: Alle Befragten $(n=28.300)$.

In der Tat ist bei hohem Haushaltsnettoeinkommen nicht nur der Anteil der Personen höher, die überhaupt Geld spenden, sondern auch der Anteil der Personen, die große Summen spenden. Sind es bei den Personen aus Haushalten mit bis zu 1.000 Euro monatlichem Haushaltsnettoeinkommen lediglich 36,7 Prozent, die Geld spenden, so finden sich unter den Personen aus Haushalten mit den höchsten Haushaltsnettoeinkommen (mit mehr als 4.000 Euro monatlich) 71,6 Prozent Geldspenderinnen und Geldspender (Abbildung 7-3). Zudem ist der Anteil von Personen, die im Zeitraum eines Jahres über 1.000 Euro spenden, mit 7,7 Prozent am höchsten bei Personen mit sehr guter Einkommensposition von über 4.000 Euro monatlichem Haushaltsnettoeinkommen. Der Zusammenhang zwischen Haushaltsnettoeinkommen und Geldspenden zeigt sich somit sowohl bezüglich der Fragen ob, als auch insbesondere wie viel Personen spenden.

Dieses deutliche Ergebnis steht im Einklang mit Befunden auf Basis des SOEP, die zeigen, dass Besserverdienende nicht nur in absoluten Beträgen, sondern auch anteilig an ihrem Monatseinkommen mehr spenden als Personen mit geringerem Einkommen (Priller \& Schupp
2011). Offensichtlich ist aber auch, dass die Bereitschaft Geld zu spenden, nicht allein durch die Einkommenssituation bestimmt ist. Denn auch Personen, die eher als armutsgefährdet eingeschätzt werden können (mit einem monatlichen Einkommen von bis zu 1.000 Euro), weisen einen vergleichsweise hohen Spendenanteil von 36,7 Prozent auf. ${ }^{1}$ Die unvorteilhaftere ökonomische Situation drückt sich hier weniger in der allgemeinen Spendenbereitschaft aus, sondern lässt sich eher an der Höhe der Spendenbeträge ablesen: Es gibt in der untersten Einkommensgruppe erwartungsgemäß kaum Personen, die Beträge von 500 Euro und mehr pro Jahr spenden. 28,0 Prozent der Personen, die in einem Haushalt leben, dem monatlich weniger als 1.000 Euro zur Verfügung stehen, haben in den vergangenen zwölf Monaten bis zu 100 Euro gespendet.

1 Die Armutsschwelle lag 2014 bei 987 Euro pro Monat für einen Einpersonenhaushalt. Bei einem Haushaltseinkommen unterhalb dieses Schwellenwertes ist von einer Armutsgefährdung des Haushaltes auszugehen (Statistisches Bundesamt 2016). 


\subsection{Geldspenden und Spendenbeträge im Zeitvergleich}

Im Zeitvergleich hat der Anteil der Geldspenderinnen und -spender an der Wohnbevölkerung Deutschlands abgenommen: 1999 betrug der Anteil noch 63,4 Prozent, 2004 lag er bei 63,9 Prozent, 2009 betrug er 58,2 Prozent, und 2014 spendeten mit 54,4 Prozent nur noch etwas mehr als die Hälfte aller Personen im Alter ab 14 Jahren (Abbildung 7-4). Die Gründe für den Rückgang der Spendenquote können vielfältig sein. Sie können sowohl auf der Seite der Organisationen liegen - hier gibt es oft Kritik an Organisationen, die einen zu großen Teil der Spenden für die Verwaltung verwenden statt für konkrete Projekte -, als auch auf der Seite der Gesellschaft und der Individuen - eventuell drückt sich die steigende soziale Ungleichheit bezüglich der Einkommensverteilung und der steigenden Armutsquoten eben auch in der nachlassenden Spendenbereitschaft aus. Im Folgenden betrachten wir deshalb, bei welchen Bevölkerungsgruppen die Spendenanteile besonders deutlich gesunken sind.

Der zu beobachtende Rückgang der Spendenquote zeigt sich in allen Bevölkerungsgruppen: So betrug die Spendenquote der Frauen im Jahr 1999 noch 65,0 Prozent und im Jahr 2014 nur noch 57,2 Prozent. Bei den Männern ist die Spendenquote im gleichen Zeitraum von 61,5 Prozent (1999) auf 51,5 Prozent (2014) gesunken (Abbildung 7-5a).

\section{Abbildung 7-4: Anteile von Personen, die Geld spenden, im Zeitvergleich}

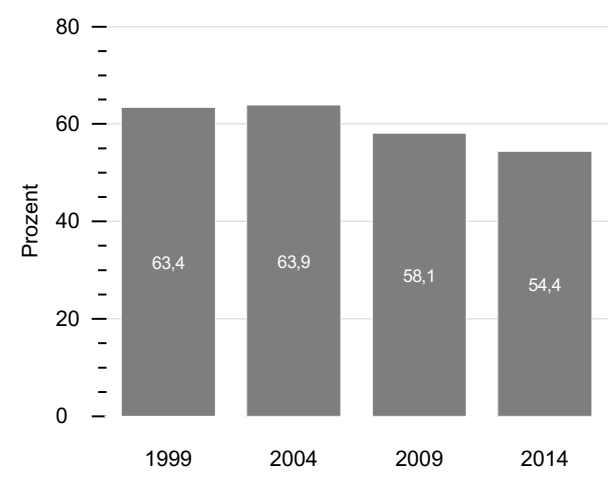

Quelle: FWS, gewichtet, eigene Berechnungen (DZA). Basis: Alle Befragten. FWS 1999 ( $n=14.795)$, FWS 2004 $(n=14.946)$. FWS $2009(n=19.964)$, FWS $2014(n=28.574)$. 
Abbildung 7-5: Anteile von Personen, die Geld spenden, im Zeitvergleich, a) nach Geschlecht, b) nach Alter und c) nach Bildung

\section{a) nach Geschlecht}

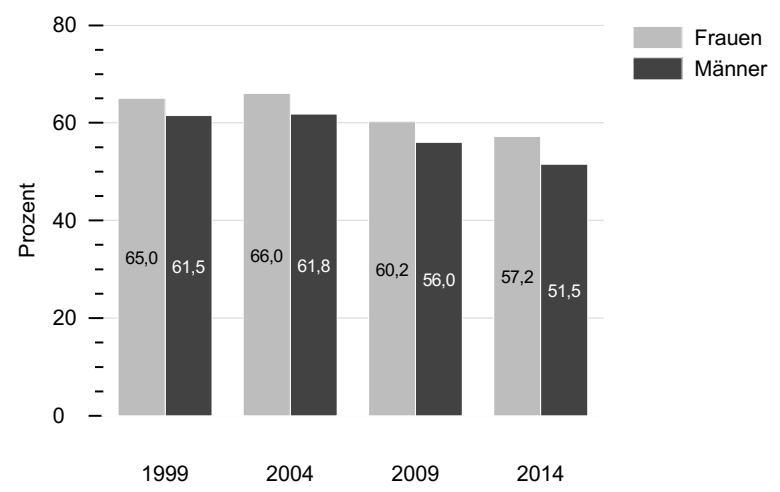

b) nach Alter

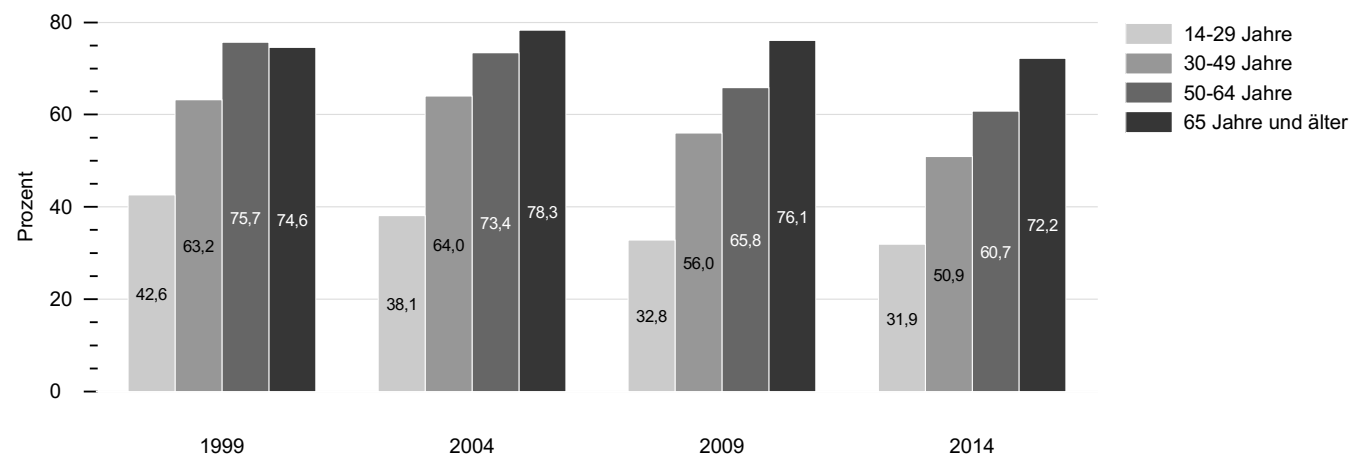

c) nach Bildung

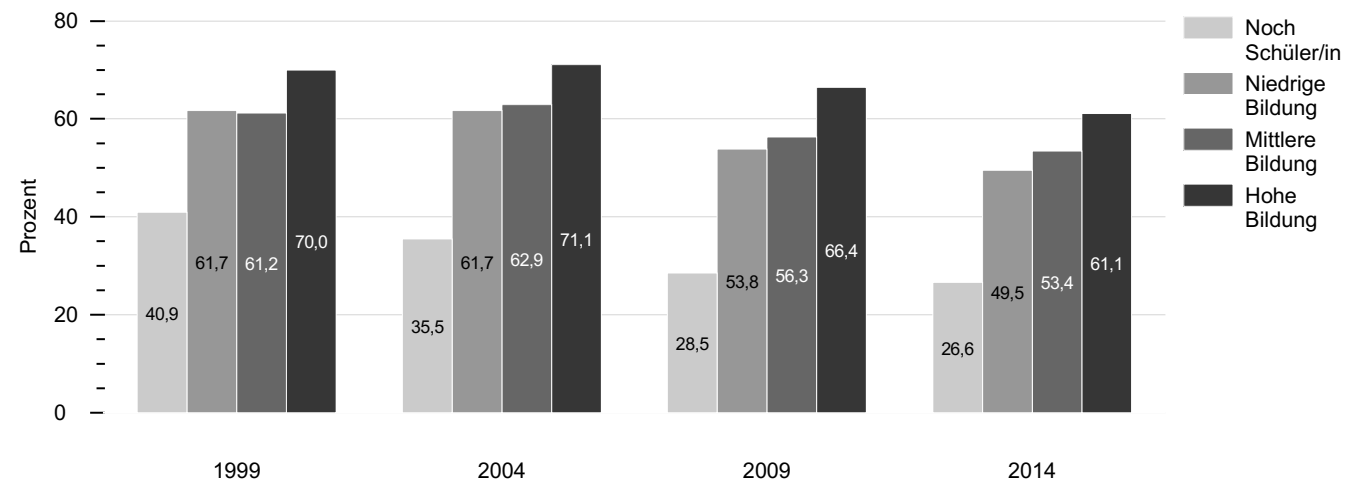

Quelle: FWS, gewichtet, eigene Berechnungen (DZA). Basis: Alle Befragten. FWS $1999(n=14.795)$, FWS 2004 $(n=14.946)$. FWS $2009(n=19.964)$, FWS $2014(n=28.574)$. 
Auch in allen Altersgruppen ist dieser Trend $\mathrm{zu}$ beobachten (Abbildung 7-5b): In der Altersgruppe der 14- bis 29-Jährigen ist die Spendenquote von 42,6 Prozent (1999) auf 31,9 Prozent (2014) gesunken, in der Altersgruppe der 30- bis 49-Jährigen ebenso deutlich von 63,2 Prozent (1999) auf 50,9 Prozent (2014). Besonders groß ist die Differenz auch in der Altersgruppe der 50 - bis 64-Jährigen, sie ist von 75,7 Prozent - das war die höchste altersgruppenspezifische Quote im Jahr 1999 - auf 60,7 Prozent im Jahr 2014 gesunken. Lediglich in der Altersgruppe der Personen im Alter von 65 und mehr Jahren ist der Einbruch nicht so stark, mit einer geringen Abnahme von 74,6 Prozent (1999) auf 72,2 Prozent (2014). Das könnte damit zusammenhängen, dass die Einschnitte bei der Altersvorsorge aufgrund der schrittweisen Anhebung der Regelaltersgrenze auf 67 Jahren und dem Absenken des Leistungsniveaus in der gesetzlichen Rentenversicherung weniger Personen betreffen, die bereits im Ruhestand sind, als vielmehr die jüngeren, noch erwerbstätigen Personen treffen werden, die in den nächsten beiden Jahrzehnten in den Ruhestand treten.
Darüber hinaus geht auch in allen vier in unserer Analyse betrachteten Bildungsgruppen die Spendentätigkeit zurück (Abbildung 7-5c): 1999 haben von den Personen mit niedriger Bildung noch 61,7 Prozent Geld gespendet, 2014 waren es nur 49,5 Prozent. Bei den Personen mit hoher Bildung ist die Spendenquote im gleichen Zeitraum von 70,0 Prozent auf 61,1 Prozent gesunken. Am deutlichsten ist der Rückgang jedoch bei den Schülerinnen und Schülern.

Betrachtet man die gespendeten Beträge im Zeitvergleich, zeigt sich insbesondere, dass der Anteil der Personen, die kleine Beträge spenden, gesunken ist: Waren es im Jahr 1999 noch 41,4 Prozent aller Personen, die bis zu 100 Euro pro Jahr spendeten, sank dieser Anteil auf 37,0 Prozent im Jahr 2004, auf 33,4 Prozent im Jahr 2009, und schließlich auf 31,3 Prozent im Jahr 2014 (Abbildung 7-6). Verschärft wird diese Entwicklung noch durch den Kaufkraftverlust aufgrund von Inflation. Die Menschen spenden nicht nur seltener bis zu 100 Euro, sondern faktisch lässt sich mit diesem Betrag seitens der Empfängerorganisationen heute auch weniger erreichen als beispielsweise noch vor zehn Jahren.

Abbildung 7-6: Anteile von Personen, die Geld spenden, im Zeitvergleich, differenziert nach gespendeten Beträgen

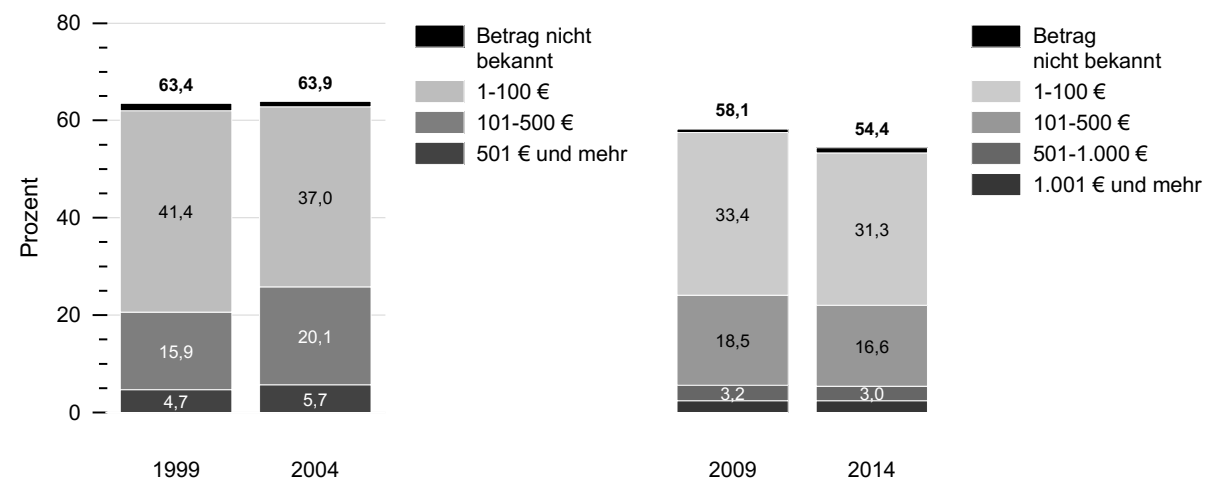

Quelle: FWS, gewichtet, eigene Berechnungen (DZA). Basis: Alle Befragten. FWS 1999 ( $n=14.589$ ), FWS 2004 $(n=14.830)$. FWS $2009(n=19.852)$, FWS $2014(n=28.300)$. 
Bei den höheren Spendenbeträgen von über 500 Euro zeigt sich dagegen Stabilität: Im Jahr 2004 spendete in Deutschland ein Anteil von 5,7 Prozent aller Bürgerinnen und Bürger eine Summe von mindestens über 500 Euro pro Jahr, dieses Niveau hält sich stabil in den folgenden zehn Jahren (6,2 Prozent 2009; 6,5 Prozent 2014). Die größten Spenden mit Beträgen über 1.000 Euro sind jedoch nach wie vor sehr selten.

Über die gespendeten Beträge lässt sich im Zeitvergleich zwar aussagen, dass der Gesamtanteil von Personen, die kleine Beträge von bis $\mathrm{zu}$ 100 Euro spenden, über die vergangenen fünfzehn Jahre tendenziell zurückgegangen ist, während der Anteil von Personen, die große Beträge spenden stabil erscheint. Betrachtet man jedoch ausschließlich die Geberinnen und Geber, ergibt sich folgendes Bild: Von allen Geberinnen und Gebern haben 66,8 Prozent im Jahr 1999 bis zu 100 Euro gespendet, 2014 sind es 58,7 Prozent.
Beträge über 500 Euro hat 1999 ein Anteil von 7,5 Prozent gespendet, 2014 sind es 10,2 Prozent (ohne Abbildung). Das heißt, insgesamt spenden heute zwar anteilig weniger Personen, dieser Rückgang lässt sich aber bei den höheren Spendenbeträgen nicht verzeichnen. Deshalb erscheinen die Personen, die heute spenden, in der Tendenz großzügiger als die Spenderinnen und Spender in der Vergangenheit. Aufgrund der nach oben offenen Erhebungskategorie von 1.000 Euro und mehr haben wir keine Information darüber, ob sich auch innerhalb der Gruppe der großen Spendenbeträge entsprechende Veränderungen zeigen. In dieser Kategorie können sich sehr unterschiedlich hohe Geldbeträge verbergen. Dass Privatpersonen auch sehr große Beträge geben, die ein Vielfaches der im Freiwilligensurvey erfragten Spendenbeträge ausmachen, ist möglich, aber selten. ${ }^{2}$

\subsection{Spendenvolumen 2009 und 2014 im Vergleich}

Das Spendenvolumen lässt sich auf Basis des Freiwilligensurveys 2014 konservativ geschätzt auf 5,9 Milliarden Euro beziffern. Zu diesem Ergebnis kommen wir, indem wir die Spendenanteile erstens auf die Bevölkerung hochrechnen und zweitens die genannten Kategorien der Spendenbeträge berücksichtigen: ${ }^{3}$ Der Anteil (54,4 Prozent) von Spenderinnen und Spender an der Wohnbevölkerung Deutschlands im Alter ab 14 Jahren (70,9 Millionen) entspricht einer Bevölkerungsgruppe von rund 38,6 Millionen Menschen.

Im Jahr 2014 haben davon 22,2 Millionen bis einschließlich 100 Euro gespendet. Für diese Personen rechnen wir mit einem durchschnittlichen Spendenbetrag von 20 Euro (entspricht dem ersten Fünftel der Kategorienspannweite von 100 Euro) und kommen auf ein Volumen von 0,444 Milliarden Euro (Tabelle 7-2). Weitere 11,8 Millionen haben zwischen 101 Euro bis einschließlich 500 Euro gespendet, für diese Personen rechnen wir mit einem durchschnittlichen Spendenbetrag von 180 Euro, sodass sich ein Volumen von 2,118 Milliarden ergibt. Weitere 2,1 Millionen haben bis einschließlich 1.000 Euro gespendet, für diese Personen rechnen wir mit einem durchschnittlichen Spendenbetrag von 600 Euro. Weitere 1,7 Millionen haben über 1.000 Euro gespendet. Wir rechnen für diese Personen mit einem durchschnittlichen Spendenbetrag von 1.200 Euro. Von 0,8 Millionen Menschen wissen wir, dass sie gespendet haben, aber nicht, in welcher Höhe, da hier die Befragten im Freiwilligensurvey den Betrag nicht nennen wollten oder konnten. Für

2 Als Beispiel: The Giving Pledge ist eine Kampagne von Bill Gates und Warren Buffet, die reiche Familien auffordern, die Hälfte ihres Vermögens zu spenden. http://givingpledge.org

3 Ein solches Verfahren zur Schätzung des gesellschaftlichen Gesamtvolumens wird beispielsweise auch für die Darstellung des Gesamtvolumens von Vermögensübertragungen durch Erbschaften oder durch Schenkungen verwendet (Lux \& Schupp 2010; Kohli \& Schupp 2005). 
diese Personen rechnen wir ebenfalls mit einem durchschnittlichen Spendenbetrag von 20 Euro, weil die Spendenkategorie bis 100 Euro am häufigsten genannt wird. Summieren wir die Volumen auf, so ergibt sich für 2014 ein geschätztes Gesamtspendenvolumen von 5,896 Milliarden (Tabelle 7-2). Diese Schätzung erscheint auch im Vergleich zu den Schätzungen des Spendenvolumens aus anderen Datenquellen wie der Einkommensteuerstatistik plausibel.

Vergleichen wir das auf diese Weise geschätzte Spendenvolumen aus 2014 mit dem nach dem selben Verfahren geschätzten Spendenvolumen aus 2009, sehen wir, dass das Gesamtspendenvo- lumen leicht zurückgegangen ist, und zwar von 6,248 Milliarden Euro auf 5,896 Milliarden Euro (Tabelle 7-2). Der Rückgang ist auf einen Rückgang des Anteils der Spenderinnen und Spender von 58,2 Prozent im Jahr 2009 auf 54,4 Prozent im Jahr 2014 zurückzuführen, der insbesondere zu einem Rückgang bei den kleinen und mittleren Spendenbeträgen geführt hat. Unsere Schätzung basiert auf der Annahme, dass die Wohnbevölkerung im Alter ab 14 Jahren sich im Zeitraum von fünf Jahren nicht verändert hat. 2009 lag somit nicht nur die Spendenquote, sondern auch das Spendenvolumen etwas höher als 2014.

Tabelle 7-2: Spendenvolumen 2009 und 2014

\begin{tabular}{lcccc}
\hline & $\begin{array}{c}\text { Menschen } \\
\text { Anzahl } \\
\text { in Millionen }\end{array}$ & $\begin{array}{c}\text { Volumen } \\
\text { Beträge } \\
\text { in Milliarden Euro }\end{array}$ & $\begin{array}{c}\text { Menschen } \\
\text { Anzahl } \\
\text { in Millionen }\end{array}$ & $\begin{array}{c}\mathbf{2 0 1 4} \\
\text { Volumen } \\
\text { Beträge } \\
\text { in Milliarden Euro }\end{array}$ \\
\hline 1 bis 100 Euro & 23,7 & 0,474 & 22,2 & 0,444 \\
\hline 101 bis 500 Euro & 13,1 & 2,361 & 11,8 & 2,118 \\
\hline 501 bis 1.000 Euro & 2,3 & 1,361 & 2,1 & 1,276 \\
\hline Über 1.000 Euro & 1,7 & 2,042 & 1,7 & 2,042 \\
\hline Spendenbetrag unbekannt & 0,5 & 0,010 & 0,8 & 0,016 \\
\hline Keine Spende & 29,6 & 0,000 & 32,3 & 0,000 \\
\hline Gesamt & $\mathbf{7 0 , 9}$ & $\mathbf{6 , 2 4 8}$ & $\mathbf{7 0 , 9}$ & $\mathbf{5 , 8 9 6}$ \\
\hline
\end{tabular}

Quelle: FWS, gewichtet, eigene Berechnungen (DZA). Basis: Alle Befragten. FWS 2009 ( $n=19.852$ ), FWS 2014 ( $n=28.300)$.

Nachrichtlich: Zur Berechnung des Spendenvolumens wurde für die Kategorie 1 bis 100 Euro ein durchschnittlicher Spendenbetrag von 20 Euro eingesetzt, für die Kategorie 101 bis 500 Euro ein durchschnittlicher Spendenbetrag von 180 Euro, für die Kategorie 501 bis 1.000 Euro ein durchschnittlicher Spendenbetrag von 600 Euro, für die Kategorie von über 1.000 Euro ein durchschnittlicher Spendenbetrag von 1.200 Euro. Diese Werte entsprechen jeweils der Untergrenze plus dem ersten Fünftel der jeweiligen Kategorienspannweite. Da die höchste Kategorie keine Obergrenze hat, haben wir eine hypothetische Obergrenze von 2.000 Euro bestimmt und davon jeweils wieder ein Fünftel auf die Untergrenze zugeschlagen. War der Spendenbetrag unbekannt, wurde ein durchschnittlicher Spendenbetrag von 20 Euro als typischer Wert angenommen, da diese Kategorie (bis 100 Euro) am häufigsten genannt wurde.

Alternative Szenarien mit unterschiedlichen Annahmen: Werden in einer alternativen Berechnung höhere Werte pro Kategorie angenommen (etwa die jeweiligen Kategorienmittelwerte), so erhöhen sich zwar die geschätzten Spendenvolumen pro Zeitpunkt, der Befund eines Absinkens des Spendenvolumens von 2009 auf 2014 bleibt jedoch bestehen. Wir präsentieren an dieser Stelle lediglich die Variante mit konservativer Schätzung, die aufgrund der Tatsache, dass Einkommen und Vermögen statistisch nicht normalverteilt sind, plausibel erscheint. Einkommen und Vermögen sind rechtsschief verteilt, das heißt, viele Menschen besitzen sehr kleine Geldbeträge, aber nur wenige Menschen besitzten große oder sehr große Geldbeträge. 


\subsection{Geldspenden und freiwilliges Engagement}

Personen, die Geld spenden, sind zu höheren Anteilen freiwillig engagiert als Personen, die kein Geld spenden. Frauen, die in den vergangenen zwölf Monaten Geld gespendet haben, enga- gieren sich mit 46,7 Prozent häufiger als Frauen, die nicht gespendet haben (34,7 Prozent), bei Männern liegen die entsprechenden Anteile bei 52,1 Prozent und 39,2 Prozent (Abbildung 7-7).

\section{Abbildung 7-7: Anteile Engagierter 2014, nach Geldspende, a) gesamt, nach Geschlecht und nach Alter, b) nach Bildung}

a) gesamt, nach Geschlecht und nach Alter

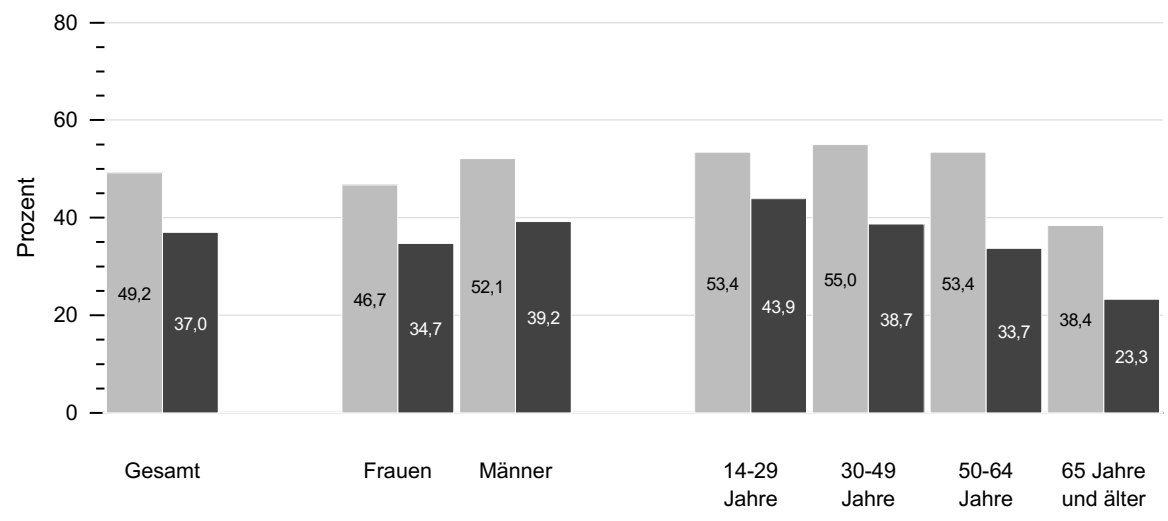

\section{b) nach Bildung}

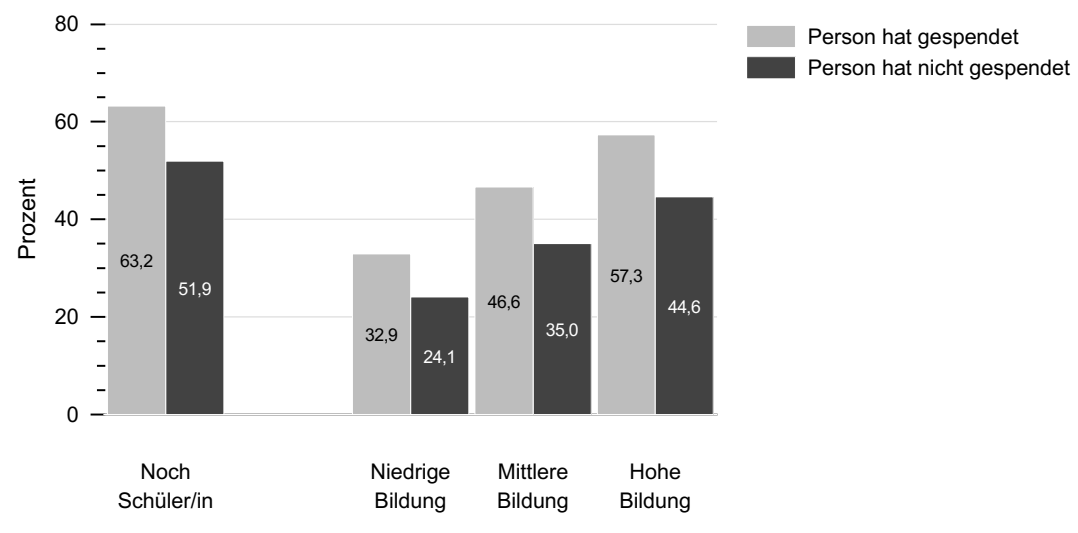

Quelle: FWS 2014, gewichtet, eigene Berechnungen (DZA). Basis: Alle Befragten $(n=28.574)$.

Die Unterschiede sind insbesondere in den mittleren Altersgruppen stark ausgeprägt, hier werden Geldspenden und Engagement offenbar häufiger miteinander kombiniert als bei den 14- bis 29-Jährigen. Die Unterschiede zeigen sich in allen Bildungsgruppen, wenn auch auf 
unterschiedlichem Niveau. Da der Anteil Engagierter in allen Bevölkerungsgruppen höher ist bei denjenigen, die Geld spenden, als bei denen, die nicht spenden, ist davon auszugehen, dass sich Geldspenden und freiwilliges Engagement ergänzen, und es sich nicht um konkurrierende Verhaltensweisen handelt.

\subsection{Fazit}

Mehr als die Hälfte der Bevölkerung im Alter ab 14 Jahren spendet Geld (54,4 Prozent). Die Spendenquote liegt damit deutlich über dem Anteil von Personen, die sich freiwillig engagieren (43,6 Prozent). Zudem liegt die im Deutschen Freiwilligensurvey ausgewiesene Spendenquote in der Tendenz etwas höher als die aus anderen Datenquellen wie etwa dem SOEP (Deutscher Bundestag 2012: 97; Priller \& Schupp 2010). Die Anlässe, um für eine bestimmte Sache oder ein bestimmtes Ziel oder eine bestimmte Organisation zu spenden sind vielfältig und zahlreich, und sie nehmen möglicherweise sogar $\mathrm{zu}$, weil die weltweite Berichterstattung auch Naturkatastrophen, Krisen und Krankheiten in anderen Teilen der Welt ins globale Bewusstsein rückt. Die Anlässe im Einzelnen kennen wir nicht, aber ein paar Beispiele zeigen bereits, wie vielfältig die Spendenanlässe sind: Im Jahr 2014 gab es etwa Spendenaufrufe zur Elbe-Flut und Donau-Überschwemmung, zu den schweren Erdbeben in Japan und China, bezüglich der Ebola-Epidemie in Westafrika und den Flüchtlingsströmen aus Syrien. Warum aber spenden manche Menschen und andere nicht?

Neben einer Organisation oder Einrichtung, an die eine Geldspende fließen soll, setzt das Spenden bei den Geberinnen und Gebern eine minimale finanzielle Fähigkeit zum Spenden voraus. Unsere Ergebnisse stehen im Einklang mit Befunden anderer Studien, welche die deutlichen Zusammenhänge zwischen Einkommen und Spendentätigkeit sowie zwischen Einkommen und Spendenhöhe ebenfalls belegt haben (beispielsweise Priller \& Schupp 2011). Offensichtlich spenden aber auch Personen, die finanziell nicht gut gestellt sind. Verschiedene Motive zu geben, etwa sich gut zu fühlen, wenn man etwas Sinnvolles getan hat oder jemandem helfen konnte, Einfluss nehmen zu wollen oder soziale Erwartungen von Verwandten oder Bekannten zu erfüllen, spielen hierbei eine zentrale Rolle. Die solidarischen Werte und Normen zum Spenden sind teils auch religiös verankert. Aus der Literatur ist zum Beispiel bekannt, dass Personen, die einer Konfession angehören anteilig häufiger spenden als Konfessionslose, da im Christentum und im Islam wie auch in anderen Weltreligionen das Spenden (auch Almosen in der katholischen Kirche oder Zakat in islamischen Religionsgemeinschaften) zur Unterstützung der Armen als Bestandteil der Religionsausübung angesehen wird (Bekkers \& Wiepking 2011; 2012). Ob jemand Geld spendet oder nicht, ist nicht allein das Ergebnis einer rein finanziellen Abwägung, sondern auch die Folge der Abwägung moralischer Verpflichtungen und gesellschaftlicher Solidarität. Menschen spenden zudem aus sozialen beziehungsweise karitativen Gründen, weil sie helfen wollen, möglicherweise auch, weil diese Hilfe von ihnen auch aus religiösen oder solidarischen Erwägungen erwartet wird, oder weil sie etwas zurückgeben wollen. Sie spenden auch höhere Beträge, wenn ihre finanziellen Mittel es ihnen erlauben. Es gibt also eine Vielzahl von Motiven und Einflussfaktoren, warum Menschen Geld spenden. West (2011) hat in ihrer qualitativen Studie verschiedene Typen von Geldspenderinnen und Geldspendern herausgearbeitet, an denen die Vielschichtigkeit der Motive für Spenden verdeutlicht werden kann: Manche finden es selbstverständlich, den Schwächeren in der Gesellschaft zu helfen, andere wollen politisch Einfluss nehmen und konkrete Ziele unterstützen. Darüber hinaus gibt es laut West (2011) Personen, die in Reaktion auf emoti- 
onale Anreize handeln, ohne konkrete politische Zielstellung. Auch die Frage, warum Menschen in Deutschland heute zu geringeren Anteilen spenden als früher, ist nicht eindimensional $\mathrm{zu}$ beantworten (Lingelbach 2010).

Wie lässt sich der Rückgang der Spendenquote bei Geldspenden von neun Prozentpunkten im Zeitvergleich deuten? Noch spendet mehr als jeder beziehungsweise jede Zweite in der Bevölkerung, trotzdem könnte der stetige Rückgang der Spendenquote auf Prozesse einer teilweisen Entsolidarisierung in bestimmten Bevölkerungsgruppen hindeuten. Festzuhalten ist aber auch, dass sich die Spendenquote gegenläufig zur Engagementquote entwickelt. In den vergangenen fünfzehn Jahren ist der Anteil der Personen gestiegen, die sich freiwillig engagieren, der Anteil der Personen aber zurückgegangen, die Geld spenden. Dieser gegenläufige Entwicklungsprozess scheint auf den ersten Blick paradox, insbesondere vor dem Hintergrund, dass eine bessere individuelle Ressourcenausstattung - etwa hohe Bildung - sowohl Engagement als auch Geldspenden begünstigt und Personen, die Geld spenden, sich auch überdurchschnittlich häufig freiwillig engagieren, da Spenden und Engagement einander ergänzende Verhaltensweisen darstellen. Auf den zweiten Blick deutet diese pa- radox erscheinende Entwicklung möglicherweise auf eine Verschiebung der Spendenstrategien der Bürgerinnen und Bürger hin. Statt Geld zu spenden, ohne tatsächlich transparent und offen nachvollziehen und kontrollieren zu können, wie dieses verwendet wird, tendieren heute offensichtlich mehr Menschen dazu, Zeit zu spenden. Das heißt, sie bringen ihre Arbeitskraft ein und übernehmen selbst freiwillige Tätigkeiten und die damit einhergehende soziale Verantwortung. Vielleicht tendieren sie auch dazu, eher Sachen zu spenden, die gebraucht werden, als Geld. Schließlich liegt die Intention zu helfen und mitzugestalten gleichermaßen sowohl dem Spenden von Geld als auch dem Spenden von Zeit oder dem Spenden von Sachen zugrunde. Verändert hat sich vielleicht die Einschätzung der Bürgerinnen und Bürger, welche Art und Weise zu helfen und mitzugestalten die zielführendere ist. Darüber hinaus ist denkbar, dass es einen steigenden Anteil an Personen gibt, die sich aufgrund ihrer Einkommens- und Vermögenssituation nicht mehr in der Lage sehen, ihre knappen finanziellen Ressourcen für die Gemeinschaft zur Verfügung zu stellen, dies allerdings durch vermehrte freiwillige Tätigkeiten kompensieren und Zeit statt Geld spenden.

\section{Literatur}

Bekkers, R., \& Wiepking, P. (2012). Who Gives? A Literature Review of Predictors of Charitable Giving. II - Gender, Family Composition and Income. Voluntary Sector Review, 3(2), 217-245.

Bekkers, R., \& Wiepking, P. (2011). Who Gives? A Literature Review of Predictors of Charitable Giving. I - Religion, Education, Age, and Socialization. Voluntary Sector Review, 2(3), 337-365.

Buschle, N. (2006). Spenden in Deutschland. Ergebnisse der Einkommensteuerstatistik 2001. In: Wirtschaft und Statistik, 2, 151-159.

Deutscher Bundestag (Hrsg.) (2012). Erster Engagementbericht - Für eine Kultur der Mitverantwortung. Bericht der Sachverständigenkommission und Stellungnahme der Bundesregierung (Bundestagsdrucksache 17/10580). Berlin: Deutscher Bundestag.

Deutsches Zentralinstitut für soziale Fragen (DZI) (2015). Spendenbilanz 2014: Allgemeine Spenden legen kräftig zu (Pressemitteilung vom 9.3.2015). Online: http://www.dzi.de/wp-content/uploads/2015/03/DZI_PM_9Mrz2015.pdf (zuletzt abgerufen 5.2.2016). 
Gesellschaft für Konsumforschung (GfK) (2015). Deutsche bleiben großzügig - Spendenaufkommen 2014 steigt erneut (Pressemitteilung vom 11.3.2015). Online: https://www.gfk.com/es-mx/insights/press-release/deutsche-bleiben-grosszuegig-spendenaufkommen-2014-steigt-erneut/(zuletzt abgerufen 1.3.2016).

Kohli, M., \& Schupp, J. (2005). Zusammenhänge und Wechselwirkungen zwischen Erbschaften und Vermögensverteilung. Gutachten für die Armuts- und Reichtumsberichterstattung der Bundesregierung. Berlin: Bundesministerium für Arbeit und Soziales.

Lengfeld, H. (2009). Die Angst der Mittelschicht vor dem sozialen Abstieg. Eine Längsschnittanalyse 1984-2007. Zeitschrift für Soziologie, 38(5), 379-398.

Lingelbach, G. (2010). „... die Hungernden zu speisen“ - Zur Entwicklung des Spendenverhaltens in Deutschland. Aus Politik und Zeitgeschichte, 60 (51-52), 29-34.

Lux, T., \& Schupp, J. (2010). Analyse des Erbschafts- und Schenkungsgeschehens. In: C. Vogel, H. Künemund \& U. Fachinger (Hrsg.) Die Relevanz von Erbschaften für die Altersicherung (DRV-Schriften 90) (S. 49-61). Berlin: Deutsche Rentenversicherung Bund.

Motel-Klingebiel, A., \& Vogel, C. (2013). Rückkehr der Altersarmut. In: A. Motel-Klingebiel \& C. Vogel (Hrsg.) Altern im sozialen Wandel: Die Rückkehr der Altersarmut (S. 13-23). Wiesbaden: Springer VS.

Priller, E., \& Schupp, J. (2011). Soziale und ökonomische Merkmale von Geld- und Blutspendern in Deutschland. DIW Wochenbericht, 78(29), 3-10.

Priller, E., \& Schupp, J. (2010). Zur Empirie des Spendens. In: A. Frank, E. Priller \& R. Graf Strachwitz (Hrsg.) Prosoziales Verhalten - Spenden in interdisziplinärer Perspektive (S. 41-63). Stuttgart: Lucius \& Lucius.

Priller, E., \& Sommerfeld, J. (2010). Spenden und ihre Erfassung in Deutschland. In: E. Priller \& J. Sommerfeld (Hrsg.) Spenden in Deutschland: Analysen - Konzepte - Perspektiven (S. 5-74). Berlin: LIT Verlag.

Statistisches Bundesamt (2016). Lebensbedingungen, Armutsgefährdung. Gemeinschaftsstatistik über Einkommen und Lebensbedingungen. Leben in Europa (European Union Statistics on Income and Living Conditions (EU-SILC)). Online: https://www.destatis.de/DE/ZahlenFakten/GesellschaftStaat/EinkommenKonsumLebensbedingungen/LebensbedingungenArmutsgefaehrdung/Tabellen/EUArmutsschwelleGefaehrdung_SILC.html (zuletzt abgerufen 9.2.2016).

Statistisches Bundesamt (2015). Bevölkerung und Erwerbstätigkeit. Haushalte und Familien. Ergebnisse des Mikrozensus. Wiesbaden: Statistisches Bundesamt.

West, C. M. (2011). Bestandsaufnahme der Bedeutung verschiedener Motivgruppen bei Spendern. Halle (Saale): MartinLuther-Universität Halle-Wittenberg.

Wilke, B. (2014). Zur Situation des deutschen Spendenwesens im Jahr 2014. BBE-Newsletter, 22.

Open Access Dieses Kapitel wird unter der Creative Commons Namensnennung 4.0 International Lizenz (http://creativecommons.org/licenses/by/4.0/deed.de) veröffentlicht, welche die Nutzung, Vervielfältigung, Bearbeitung, Verbreitung und Wiedergabe in jeglichem Medium und Format erlaubt, sofern Sie den/die ursprünglichen Autor(en) und die Quelle ordnungsgemäß nennen, einen Link zur Creative Commons Lizenz beifügen und angeben, ob Änderungen vorgenommen wurden.

Die in diesem Kapitel enthaltenen Bilder und sonstiges Drittmaterial unterliegen ebenfalls der genannten Creative Commons Lizenz, sofern sich aus der Abbildungslegende nichts anderes ergibt. Sofern das betreffende Material nicht unter der genannten Creative Commons Lizenz steht und die betreffende Handlung nicht nach gesetzlichen Vorschriften erlaubt ist, ist für die oben aufgeführten Weiterverwendungen des Materials die Einwilligung des jeweiligen Rechteinhabers einzuholen. 